\title{
Implantatentfernung nach Beckenringfraktur
}

\author{
Fabian Maria Stuby, Christoph Emanuel Gonser, Hagen Christopher Baron, \\ Ulrich Stöckle, Andreas Badke, Björn Gunnar Ochs
}

\section{Zusammenfassung}

Beckenringfrakturen gehören zu den seltenen Verletzungen. In den letzten Jahren erfolgt die Stabilisierung des hinteren Beckenrings überwiegend perkutan mittels transiliosakraler Schraubenfixation. Der vordere Beckenring wird in Abhängigkeit vom Verletzungsmuster mit Platten, Schrauben oder supraazetabulär eingebrachten Fixateur-externe-Montagen stabilisiert. Anhand der Literatur sowie der retrospektiven Aufarbeitung der eigenen Ergebnisse und Erfahrungen bei 80 Patienten mit einer Beckenring-Bund -C-Verletzung wird aufgezeigt, dass die Indikation zur Entfernung des Osteosynthesematerials streng zu stellen ist. Die Indikation ist fraglos gegeben bei Fixateur-externe-Montagen, Infektionen, Schraubenfehllagen, allergischen Reaktionen auf Implantate, kritischer Weichteilbedeckung bzw. -kompromittierung durch auftragende Implantate und bei verheilten Fraktu- ren am wachsenden Skelett. Eine individuelle Abwägung der Risiken und Nutzen ist jedoch beim asymptomatischen Patienten sowie beim aufgrund von posttraumatischen Veränderungen symptomatischen Patienten erforderlich. Hier sollte bei der Entscheidung das initiale Verletzungsmuster mitberücksichtigt werden. Wird die Indikation zur Materialentfernung nach einer Beckenringfraktur gestellt, so kann es sich um eine technisch anspruchsvolle Operation mit einer hohen Komplikationsrate handeln.

\section{Implant Removal after Pelvic Ring Injury}

Pelvic ring fractures are regarded as rare injuries. In recent years, minimally invasive sacroiliacal screw fixation has been increasingly used as a procedure for the treatment of these injuries, if the dorsal pelvic ring needs to be addressed. Treatment options for the anterior pelvic ring include plates, screws or external fixation. After reviewing the limited number of publications on this subject and our own experience with 80 patients with pelvic ring B- or C-type injuries during a period of 8 years, we can show that implant removal in the pelvic ring should be indicated with caution. In some cases like external fixation, implant associated infection, malpositioning, allergic implant reaction, critical soft tissue covering, palpable implant and consolidated juvenile fractures, implant removal is certainly indicated. In patients without symptoms and in patients with trauma-associated symptoms which are not definitely associated with implants, removal is only indicated after thorough consideration of the risks versus the benefits and after taking the initial injury pattern into account. If despite all these objections, implant removal is still indicated, it must be borne in mind that implant removal may be difficult and that there are several possible severe complications.

\section{Einleitung}

Die Behandlung instabiler Beckenfrakturen stellt für den Chirurgen bis heute eine besondere Herausforderung dar. Die definitive Versorgung des hinteren Beckenrings erfolgt derzeit in den allermeisten Fällen perkutan mittels transiliosakraler Schraubenfixation. Der vordere Beckenring wird in Abhängigkeit vom Verletzungsmuster mit Platten, Schrauben oder supraazetabulär eingebrachten Fixateur-externe-Montagen stabilisiert. Die Indikation zur Entfernung des Osteosynthesematerials wird

OP-JOURNAL 2016; 32: 130-137

(c) Georg Thieme Verlag KG Stuttgart · New York DOI http://dx.doi.org/10.1055/s-0036-1585257 in der Literatur nur selten thematisiert und zudem kontrovers diskutiert.

\section{Epidemiologie, Verletzungsmuster und Klassifikation der Beckenring- verletzung}

Verletzungen des Beckenrings zählen mit einer Inzidenz von 3-20/100000 zu den seltenen Verletzungen [17]. Von allen verunfallten Patienten erleiden 3$8 \%$ eine Beckenverletzung [11], wobei leichte Verletzungen ohne Beeinträchtigung der knöchernen Stabilität des Beckenrings überwiegen [26]. Besondere Bedeutung haben instabile Beckenverletzungen, die häufig Teil eines Polytraumas sind und mit pelvinen Massenblutungen als primärer Todesursache (Letalitätsrate 5-20\% [15]) einhergehen können. Aufgrund der Verletzungs- schwere stellen diese Beckenringfrakturen einen hohen Anspruch an die Diagnostik und Versorgung.

Beckenringfrakturen werden entsprechend dem ABC-Schema nach Müller, das auch von der AO/ASIF übernommen wurde, eingeteilt (Abb.1). Bei A-Verletzungen bleibt die knöcherne und ligamentäre Integrität des dorsalen Beckenrings unangetastet. Es handelt sich daher um stabile Frakturen. B-Verletzungen führen zu einer partiellen Instabilität des Beckens bedingt durch einen Außenoder Innenrotationsverletzungsmechanismus. Die C-Verletzungen sind gekennzeichnet durch eine komplette 3-dimensionale Instabilität des Beckenrings. 


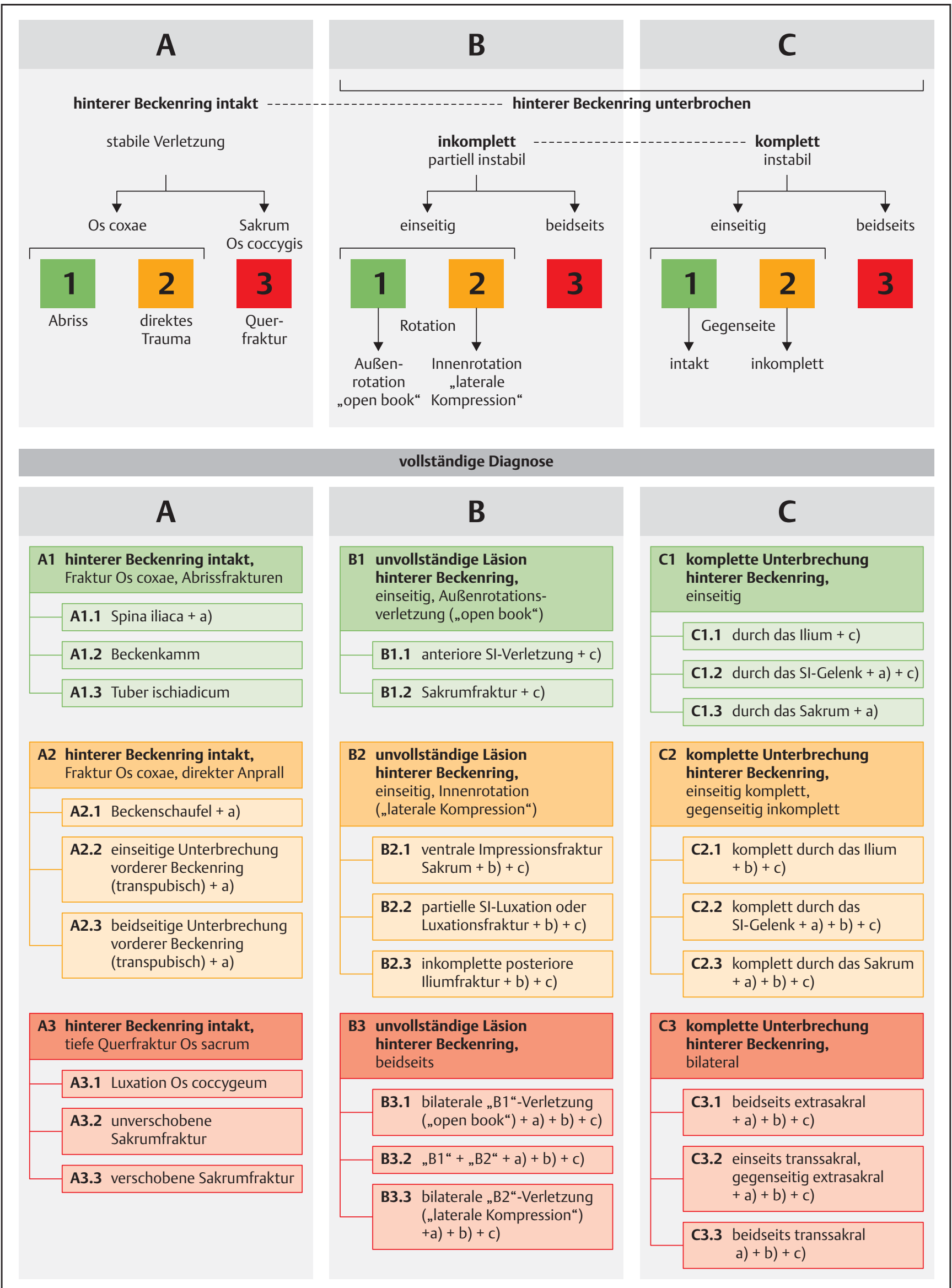

Abb. 1 CCF-Klassifikation der Beckenringverletzungen (aus [27]).

Fabian Maria Stuby et al.: Implantatentfernung nach Beckenringfraktur 


\section{Aktuelle Versorgungsstrategien des vorderen und hinteren Beckenrings}

Das primäre Therapieziel bei durch die Beckenverletzung hämodynamisch instabilen Patienten ist die Notfallstabilisierung des Beckens zur Reduktion pelviner Blutungen mithilfe der Beckenzwinge oder Fixateur-externe-Montagen. Die definitive möglichst anatomische Rekonstruktion erfolgt zumeist in der sekundären Operationsphase [13].

Eine B-Verletzung des Beckenrings wird bei einer vorliegenden Dislokation operativ behandelt. Der Eingriff lässt sich dabei auf die Fixation der ventralen Instabilität beschränken. So wird z. B. eine Symphysensprengung mit klaffender Symphyse $>2 \mathrm{~cm}$ häufig über einen Pfannenstiel-Schnitt offen reponiert und mit einer von kranial auf den Schambeinästen aufliegenden 4-Loch-Platte versorgt [28]. C-Verletzungen des Beckenrings stellen aufgrund ihres biomechanischen Stabilitätsverlusts generell eine Indikation zur operativen Stabilisierung dar. Es sollte zunächst die dorsale Instabilität versorgt werden, da sich die anschließende Reposition des ventralen Beckenrings dann einfacher gestaltet. Für die definitive Versorgung des hinteren Beckenrings eignen sich dorsal oder ventral platzierte Platten, Gewindebolzen oder transiliosakrale Schrauben [9,27]. In den letzten Jahren hat sich die transiliosakrale Schraubenosteosynthese v.a. durch die Verbesserung der intraoperativen Visualisierungsmöglichkeiten mit standardisierten Bildwandlerprojektionen wie auch durch den Einsatz der fluoroskopiebasierten Navigation für die Versorgung von Sakrumfrakturen, Sprengungen bzw. Luxationen des Iliosakralgelenks (ISG) sowie bei transiliakalen und transsakralen Luxationsfrakturen des ISG als minimalinvasives Standardverfahren etabliert [3,7]. Dieses Vorgehen zeichnet sich durch eine kurze Operationsdauer, geringen Blutverlust, minimales Weichteiltrauma und geringes Infektionsrisiko aus [10,21], setzt jedoch die geschlossene Reponierbarkeit der Verletzung voraus. Aus biomechanischer Sicht ist zudem zu beachten, dass Läsionen des vorderen Beckenrings in Abhängigkeit vom Verletzungsmuster mit Platten, Schrauben oder supraazetabulär eingebrachten Fixateur-externeMontagen ebenfalls stabilisiert werden müssen, da es sonst zur Schraubenlockerung kommen kann [19].

Tab. 1 Basisdaten Gesamtkollektiv mit operativ behandelter Beckenringverletzung. Basisdaten Gesamtkollektiv

\begin{tabular}{ll}
\hline Patientenanzahl (Gesamt) & 80 \\
\hline Geschlecht (M/W) & $57 / 23$ \\
\hline Alter zum Unfallzeitpunkt (Jahre) & $41,1 \pm 17,0$ (12-75) \\
\hline Art der Verletzung & \\
\hline - Frakturtyp B1/B2/B3 & $10 / 2 / 4$ \\
\hline - Frakturtyp C1/C2/C3 & $52 / 7 / 5$ \\
\hline Gesamtverletzungsschwere (ISS) & $25,6 \pm 10,9$ (9-57) \\
\hline Notfallmaßnahme am Unfalltag (Patienten) & 12 \\
\hline - Fixteur externe/Beckenzwinge & $8 / 4$ \\
\hline Zeit bis zur definitiven Versorgung (Tage) & $8,0 \pm 6,7(0-30)$ \\
\hline Gesamtbeobachtungszeitraum (Monate) & $23,3 \pm 19,7$ (6-101)
\end{tabular}

In der postoperativen klinischen Nachsorge der, wie dargestellt, versorgten Patienten drängt sich nach erfolgter Rehabilitation die Frage auf, inwieweit das einliegende Osteosynthesematerial entfernt werden soll. Jedoch wurden bislang die Indikationen zur Entfernung des Osteosynthesematerials bei Patienten mit Beckenringverletzungen in der Literatur kaum thematisiert und zudem kontrovers diskutiert $[2,4,7,8,16,18,27,30]$. So findet man zum einen die Empfehlung, gelenkübergreifende Implantate, wie z.B. Symphysenplatten oder transiliosakrale Schrauben (ISG-Schrauben), generell 6 bis 12 Monate nach der Versorgung zu entfernen $[4,7,27]$, zum anderen wird von einer routinemäßigen Entfernung des Osteosynthesematerials abgesehen [19] bzw. nur die Metallentfernung (ME) bei Komplikationen wie z.B. Schraubenbruch oder Infektgeschehen empfohlen $[1,22]$. Vor diesem Hintergrund haben sich die Autoren entschieden, neben der spärlich zur Verfügung stehenden Literatur die eigenen Ergebnisse und Erfahrungen bei Patienten mit Beckenringverletzungen zu analysieren, um eine mögliche Therapieempfehlung für die Implantatentfernung nach Beckenringverletzungen abgeben zu können.

\section{Analyse des eigenen Patientenkollek- tivs mit Beckenringverletzungen}

Alle Patienten, die aufgrund einer Beckenring-B- bzw. Beckenring-C-Verletzung im Zeitraum von Januar 2003 bis Dezember 2010 an der Klinik der Autoren operiert wurden, konnten im Hinblick auf das Verletzungsmuster, das gewählte Osteosyntheseverfahren sowie eine etwaige ME retrospektiv analysiert werden. Insbesondere wurden die Indikation für die ME sowie etwaige Komplikationen im Rahmen der ME beleuchtet. Als Grundlage für die Untersuchung dienten die vorhandenen Kranken- und Röntgenunterlagen sowie die klinikintern geführte Datenbank im Rahmen der wissenschaftlichen Mitarbeit in der Arbeitsgruppe Becken III der Deutschen Gesellschaft für Unfallchirurgie (AG Becken III der DGU). Patienten mit einem Nachuntersuchungszeitraum $<6$ Monate wurden ausgeschlossen. Entsprechend konnten insgesamt 80 Patienten identifiziert werden (Tab. 1).

Bei 16 Patienten mit einer Beckenring-BVerletzung wurde der vordere Beckenring $15 \times(12 \times$ Symphysenplatte; $2 \times$ Fixateur externe und Symphysenplatte; $1 \times$ Fixateur externe) und der hintere Beckenring $4 \times(3 \times$ ISG-Schrauben einseitig; $1 \times$ ISG-Schrauben beidseitig) osteosynthetisch stabilisiert. Bei 64 Patienten mit einer Beckenring-C-Verletzung wurde der hintere Beckenring immer (61× ISGSchrauben einseitig; $2 \times$ ISG-Schrauben beidseitig; $1 \times$ spinopelvine Fusion) und der vordere Beckenring in 55 Fällen (35× Fixateur externe; 17× Symphysenplatte; $3 \times$ Platte Schambeinast) versorgt.

Im postoperativen Verlauf wurde der Fixateur externe bei allen 38 Patienten $(40,3 \pm 19,8$ [18-121] Tage nach Operation) wieder entfernt. Die ME des intern einliegenden Osteosynthesematerials erfolgte bei 35 der 80 Patienten (44\%). Dabei wurde bei 11 Patienten $(5 \times$ Stabilisierung vorderer und hinterer Beckenring; 6x Stabilisierung nur vorderer Beckenring) das Osteosynthesematerial isoliert 
Tab. 2 Aufstellung der Indikationen zur ME intern einliegender Osteosynthesen.

Indikationen zur Entfernung von internen Osteosynthesen (n = 39 Patienten)

\begin{tabular}{lcl}
\hline & $\begin{array}{l}\text { hinterer Beckenring } \\
\mathbf{n}=\mathbf{2 4} \text { Patienten }\end{array}$ & $\begin{array}{l}\text { vorderer Beckenring } \\
\mathbf{n}=\mathbf{1 5} \text { Patienten }\end{array}$ \\
\hline tiefer Infekt & 1 & 1 \\
\hline Implantatlockerung & 1 & 2 \\
\hline Schmerzen & 11 & 0 \\
\hline Patientenwunsch & 3 & 6 \\
\hline ME bei Hernien-OP & 0 & 4 \\
\hline gelenkübergreifendes Implantat & 8 & 2
\end{tabular}

Tab. 3 Darstellung der Patientenkollektive mit und ohne Entfernung des einliegenden Osteosynthesematerials.

\begin{tabular}{lll}
$\begin{array}{l}\text { Gesamtpatientenkollektiv mit und ohne ME der intern einliegenden Osteosynthesen } \\
\text { (n= 80 Patienten) }\end{array}$ & ME & keine ME \\
\hline & 35 & 45 \\
\hline Patientenanzahl (Gesamt) & $23 / 12$ & $34 / 11$ \\
\hline Geschlecht (M/W) & $38,8 \pm 17,4(12-74)$ & $42,9 \pm 16,6(14-75)$ \\
\hline Alter zum Unfallzeitpunkt (Jahre) & & \\
\hline Art der Verletzung & $6 / 0 / 2$ & $4 / 2 / 2$ \\
\hline - Frakturtyp B1/B2/B3 & $21 / 5 / 1$ & $31 / 2 / 4$ \\
\hline - Frakturtyp C1/C2/C3 & $24,6 \pm 11,3(9-57)$ & $26,5 \pm 10,6(9-48)$ \\
\hline Gesamtverletzungsschwere (ISS) & 8 & 4 \\
\hline Notfallmaßnahme am Unfalltag (Patienten) & 8 & $1 / 3$ \\
\hline - Fixateur externe/Beckenzwinge & $7 / 1$ & $6,3 \pm 4,4(0-18)$ \\
\hline Zeit bis zur definitiven Versorgung (Tage) & $10,3 \pm 8,2(0-30)$ & $23,2 \pm 22,9(6-101)$ \\
\hline Gesamtbeobachtungszeitraum (Monate) & $23,0 \pm 14,7(6-67)$ & \\
\hline
\end{tabular}

am vorderen Beckenring (11× Symphysenplatte) entfernt. Bei 20 Patienten wurde isoliert das am hinteren Beckenring einliegende Osteosynthesematerial (18× ISG-Schrauben einseitig; 1× ISGSchrauben beidseitig; $1 \times$ spinopelvine Fusion) und bei 4 Patienten das am vorderen und hinteren Beckenring einliegende Osteosynthesematerial (4× Symphysenplatte; $4 \times$ ISG-Schrauben einseitig) entfernt. Die ME am hinteren Beckenring wurde $14,6 \pm 11,8(2,3-50,9)$ Monate nach Indexoperation durchgeführt (vorderer Beckenring: 20,2 $\pm 18,1$ [0,4-54,0] Monate). Die ME am hinteren Beckenring wurde insbesondere aufgrund der vom Patienten angegebenen Beschwerden (46\%) bzw. aufgrund der Empfehlung des Operateurs (33\%), das gelenküberbrückende Implantat zu entfernen, indiziert. Der gezielte Patientenwunsch $(40 \%)$ und die erforderliche Reoperation bei Narbenhernie (27\%) waren die wesentlichen Indikationen zur ME am vorderen Beckenring (Tab.2). Das initial vorliegende Verletzungsmuster bei den Patienten, bei denen das gelenküberbrückende Implantat die Indikation zur ME stellte, war am hinteren Beckenring eine transalare $(2 \times \mathrm{C} 1.3 \mathrm{a} 1)$ bzw. transforaminale (6× C1.3 a2) Sakrumfraktur und am vorderen Beckenring eine Symphysensprengung (2×; Abb. 2: Fallbeispiel I). Im Rahmen der ME kam es bei insg. 7 Patienten (20\%) zu Komplikationen (1× revisionspflichtiges Hämatom; $2 \times$ Harnblasenverletzung; $2 \times$ verbleibende Unterlegscheibe; $2 \times$ verbleibende Schraubenreste [Abb. 3 und 4: Fallbeispiel 2 und 3]). Die Metallentfernungen, bei denen es zu intraoperativen Komplikationen kam, wurden tendenziell später durchgeführt als die ME, bei denen es zu keinen Komplikationen kam (Zeitpunkt ME mit Komplikation: $24,4 \pm 20,5[4,3-54,0]$ Monate nach In- dexoperation; Zeitpunkt ME ohne Komplikationen: $13,9 \pm 11,0[2,3-52,7]$ Monate nach Indexoperation; $p=0,24$ ). Von der Metallentfernung am hinteren Beckenring profitierten 3 der 11 Patienten (33\%) im Hinblick auf die präoperativ angegebene Beschwerdesymptomatik. Bei den restlichen 8 Patienten (73\%) waren die Beschwerden am hinteren Beckenring durch die ME nicht zu beeinflussen (Verletzungsmuster: 2× C1.3 a1; $6 \mathrm{x}(1.3 \mathrm{a} 2)$.

Bei 45 der 80 Patienten (56\%) war das Osteosynthesematerial im Beobachtungszeitraum von durchschnittlich 23,2 (6-101) Monaten nach Stabilisierung einer Beckenring-B- bzw. -C-Verletzung noch einliegend. Die Charakteristika der Patienten mit und ohne ME der intern einliegenden Implantate sind in Tab. 3 vergleichend dargestellt. Die Patienten mit noch einliegenden Implantaten waren tendenziell älter (Alter: $\mathrm{p}=0,286$ ) und schwerer verletzt (ISS: $p=0,442$ ) als die Patienten mit ME. Am häufigsten wurde in beiden Kollektiven eine C1Verletzung diagnostiziert (mit ME: 60\%; ohne ME: 69\% aller Verletzungen). Innerhalb der C1-Verletzungen imponierte bei den Patienten mit ME der Verletzungstyp C1.3 (18 von 21 Fällen [86\%]) und bei den Patienten mit einliegendem Osteosynthesematerial der Typ C1.2 (14 von 31 Fällen [45\%]) und der Typ C1.3 (17 von 31 Fällen [55\%]). Diesen $31 \mathrm{~Pa}-$ tienten war im Rahmen der Nachsorge bei einem C1.3-Verletzungsmuster die Entfernung der gelenküberbrückenden Implantate empfohlen worden. Die Patienten lehnten die ME aufgrund ihrer Beschwerdefreiheit jedoch ab (Abb.5: Fallbeispiel IV). Den Patienten mit C1.2Verletzungsmuster und im Verlauf nativradiologisch erkennbarer posttraumatischer ISG-Arthrose rieten wir von der ME ab (6 von 14 [43\%]). Im Patientenkollektiv ohne ME lagen zum Zeitpunkt der letzten Nachuntersuchung in 14 Fällen gelenküberbrückende Implantate des vorderen Beckenrings (14× Symphysenplatte) bzw. in 39 Fällen gelenküberbrückende Implantate des hinteren Beckenrings (37x ISG-Schrauben einseitig; $2 \times$ ISG-Schrauben beidseitig) ein. Die Symphysenplatten waren in $79 \%$ (in 11 Fällen) gelockert $(6 x)$ bzw. gebrochen $(5 x)$ und in $21 \%$ (in 3 Fällen) unverändert einliegend. Die ISG-Schrauben zeigten in $28 \%$ (in 11 von 39 Fällen) diskrete und in $72 \%$ (in 28 von 39 Fällen) keine Lockerungszeichen. 

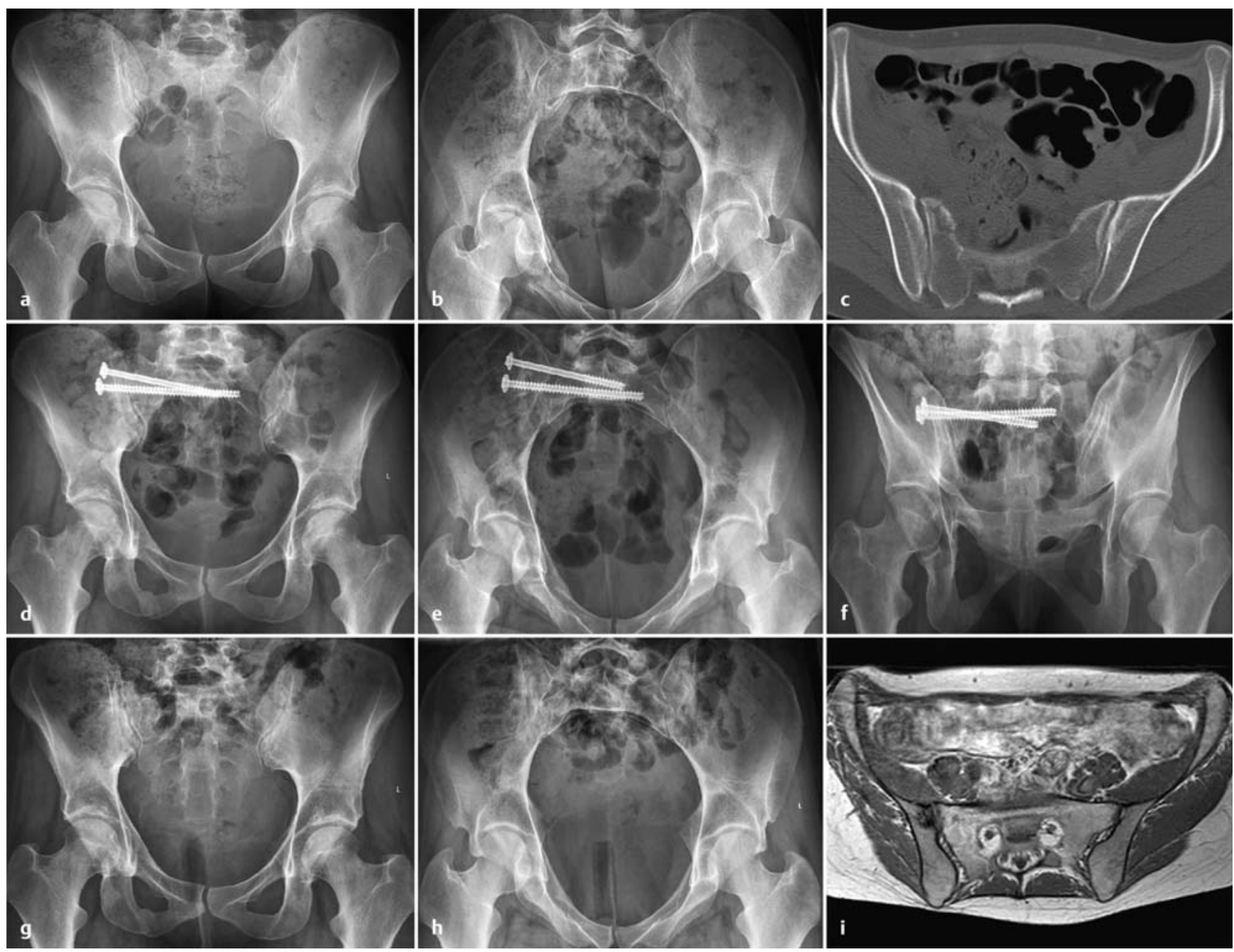

Abb. 2 a bis i Fallbeispiel I: Die Beckenringverletzung (CCF-Klassifikation C1.3 a1c1: transalare Sakrumfraktur rechts, Scham- und Sitzbeinfraktur rechts; Bildgebung am Unfalltag: a Beckenübersicht, b Inlet-Projektion, c CT Becken) einer 29-jährigen Patientin wurde dorsal mit 2 transiliosakralen Schrauben und ventral mit einem supraazetabulären Fixateur externe stabilisiert. Die Metallentfernung wurde ventral nach 7 Wochen und dorsal nach 8 Monaten durchgeführt (Bildgebung vor ME, ISG-Schrauben: d Beckenübersicht, e Inlet-Projektion, f Outlet-Projektion). Die Entfernung der transiliosakralen Schrauben wurde indiziert, um die Transfixation der ISG-Fuge aufzuheben. 12 Monate nach ME berichtete die Patientin noch über Restbeschwerden über der ISG-Fuge rechts (Bildgebung 12 Monate nach ME: g Beckenübersicht, h Inlet-Projektion, i axiale MRT-Schicht). Die MRT-Untersuchung wurde zur Beurteilung etwaiger posttraumatischer Veränderungen der ISG-Fuge durchgeführt; diese konnten bis auf eine geringfügige Deformierung des lateralen Anteils des Sakrumflügels jedoch ausgeschlossen werden.

\section{Diskussion und Empfehlung der ME am vorderen und hinteren Beckenring}

Wie oben dargestellt, besteht heutzutage Einigkeit bez. der Versorgungsstrategie von Beckenring-B- und Beckenring-CVerletzungen. Dahingegen wird die Indikationsstellung zur Materialentfernung kontrovers diskutiert $[4,7,8,16,18,27$, 30]. Insbesondere findet sich in der Literatur bislang keine ausreichende Evidenz in Form von randomisierten kontrollierten Studien mit der Fragestellung der Implantatentfernung nach Beckenringfraktur. Auch die AWMF-Leitlinie Implantatentfernung der Deutschen Gesellschaft für Unfallchirurgie bietet für die Indikationsstellung der ME bei Pa- tienten mit Beckenringverletzungen nur bedingt eine Hilfestellung [2].

Eine klare Indikation für die Implantatentfernung nach Beckenringfrakturen ist bei Fixateur-externe-Montagen [12], Infektionen [2,24], Schraubenfehllagen [9,29], allergischen Reaktionen auf Implantate $[2,6]$, kritischer Weichteilbedeckung bzw. Weichteilkompromittierung durch auftragende Implantate (z.B. einliegende spinopelvine Fusion) und bei verheilten Frakturen am wachsenden Skelett $[2,12]$ zu stellen. Ebenfalls ist es sinnvoll, im Rahmen einer erforderlichen Reoperation, wie z.B. der Versorgung einer Narbenhernie nach Stabilisierung einer Symphysensprengung mittels Plattenosteosynthese, die im selben OP-
Gebiet einliegenden Implantate zu entfernen.

Dahingegen sollten z.B. im Bereich der Schambeinäste oder der Beckenschaufel einliegende Implantate belassen werden, wenn diese nur durch aufwendige Freilegung mit entsprechend hohen zugangsbedingten Risiken entfernt werden können $[2,4,27]$. Dieser Risiken sollte sich der aufklärende Arzt bewusst sein, insbesondere dann, wenn die ME bei asymptomatischen Patienten allein aufgrund deren Wunsch zur Implantatentfernung indiziert wird. Anders verhält es sich beim symptomatischen Patienten. Hier gilt es, im Rahmen der Anamneseerhebung wie auch der klinischen und radiologischen Untersuchung $\mathrm{zu}$ 


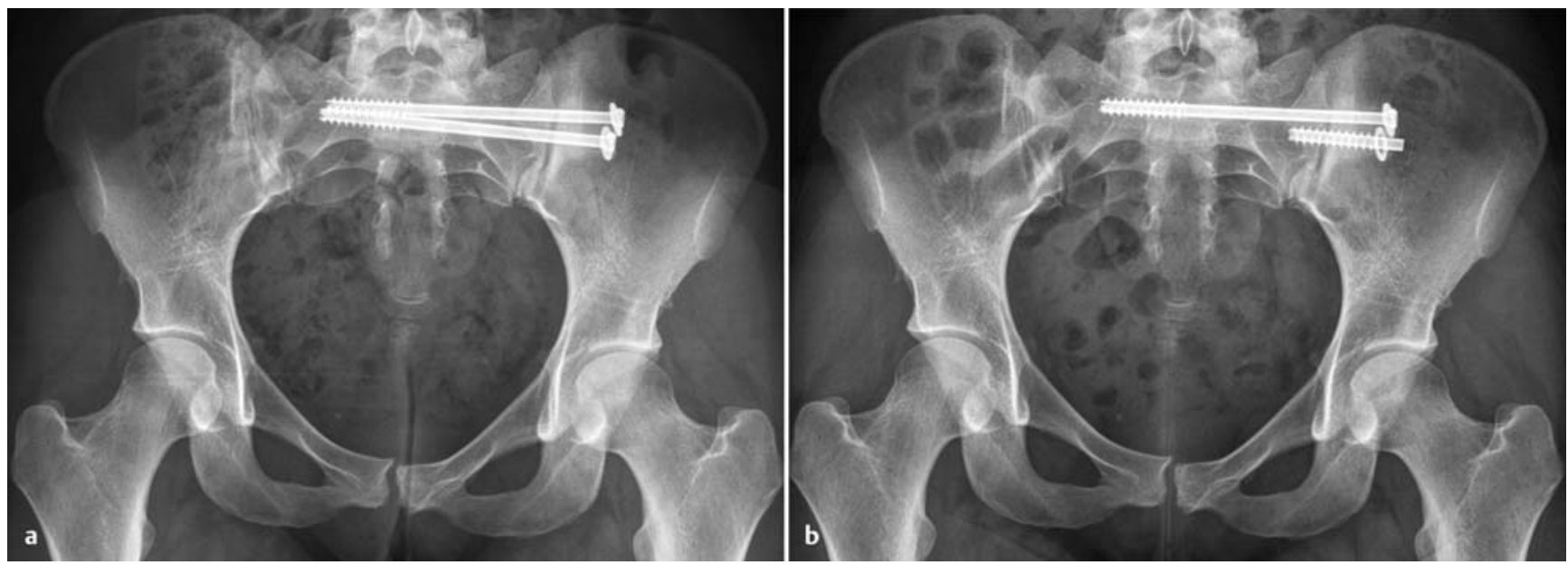

Abb. 3 a und b Fallbeispiel II: Gescheiterter Versuch der Entfernung von 2 transiliosakralen Schrauben bei einer 22-jährigen Patientin 27 Monate nach erfolgter Stabilisierung einer Beckenringfraktur (CCF-Klassifikation C1.3 a2c1: transforaminale Sakrumfraktur links, Scham- und Sitzbeinfraktur links). Die Indikation zur Metallentfernung wurde auf ausdrücklichen Wunsch der Patientin gestellt (a Beckenübersicht vor ME). Intraoperativ musste eine Schraube gekürzt werden, da ein weiteres Zurückdrehen nicht möglich war; die andere Schraube war knöchern überbrückt. Eine vollständige Entfernung wäre nur durch eine deutliche Zugangserweiterung möglich gewesen (b Beckenübersicht nach versuchter ME).
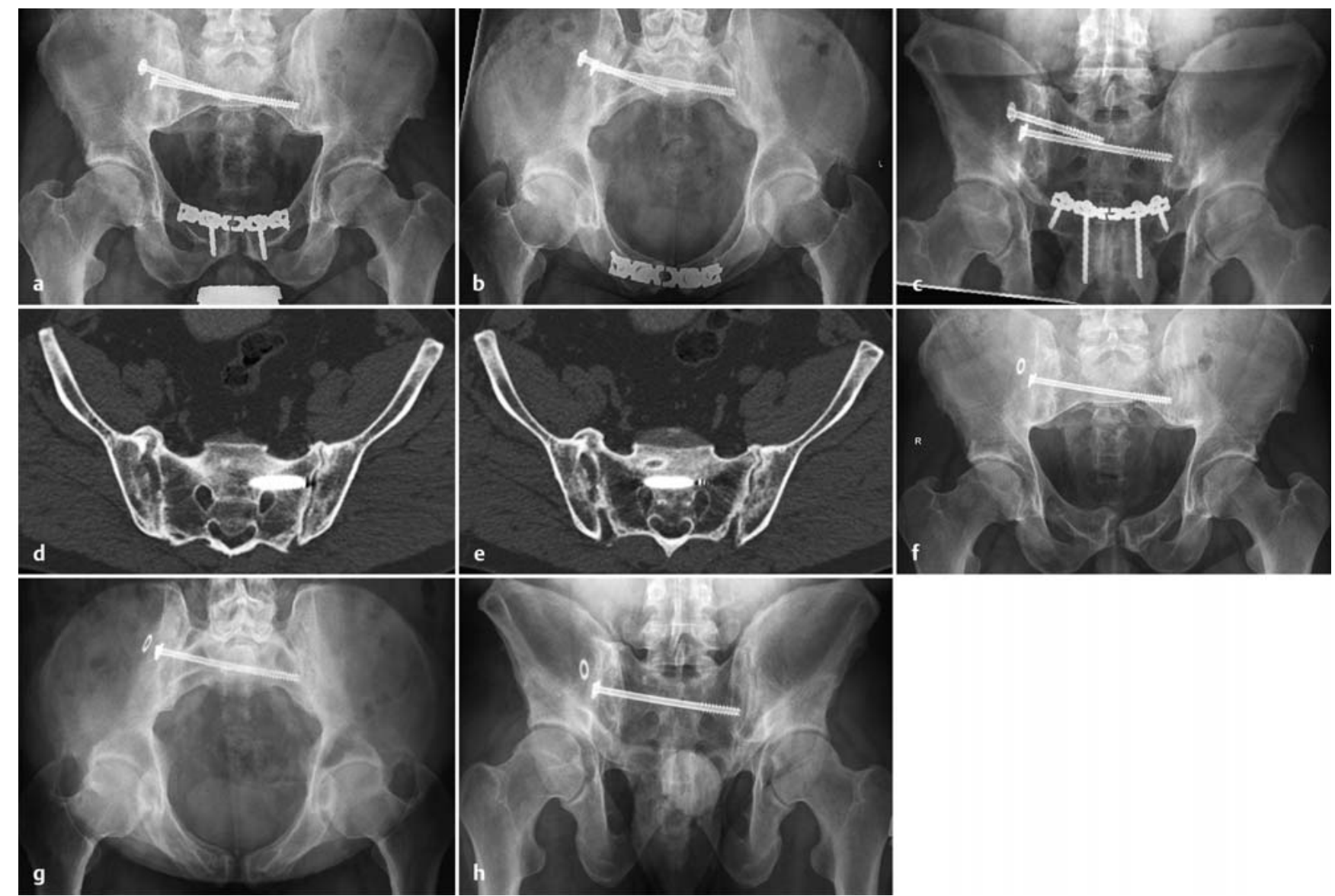

Abb. $\mathbf{4 a}$ bis $\mathbf{g}$ Fallbeispiel III: Auf ausdrücklichen Wunsch eines 35-jährigen Patienten wurde 4 Jahre nach operativer Versorgung einer Symphysenund ISG-Sprengung links (CCF-Klassifikation C1.2 a2c5) aufgrund von therapierefraktären Beschwerden insbesondere im Bereich des hinteren Beckenrings die Implantatentfernung indiziert (Bildgebung vor ME: a Beckenübersicht, b Inlet-Projektion, c Outlet-Projektion). Im Rahmen der präoperativen Vorbereitung wurde der Patient ausführlich über die fortgeschrittene Arthrose der ISG-Fuge rechts als Ursache für die Beschwerden aufgeklärt (d, e axiale CT-Schichten vor ME). Die Implantatentfernung im Bereich des hinteren Beckenrings war aufgrund der Überknöcherung der Schraubenköpfe nur z.T. möglich. Die Beschwerdesituation des Patienten konnte nicht gebessert werden (Follow-up nach ME: 14 Monate; f Beckenübersicht, $g$ Inlet-Projektion, h Outlet-Projektion). 

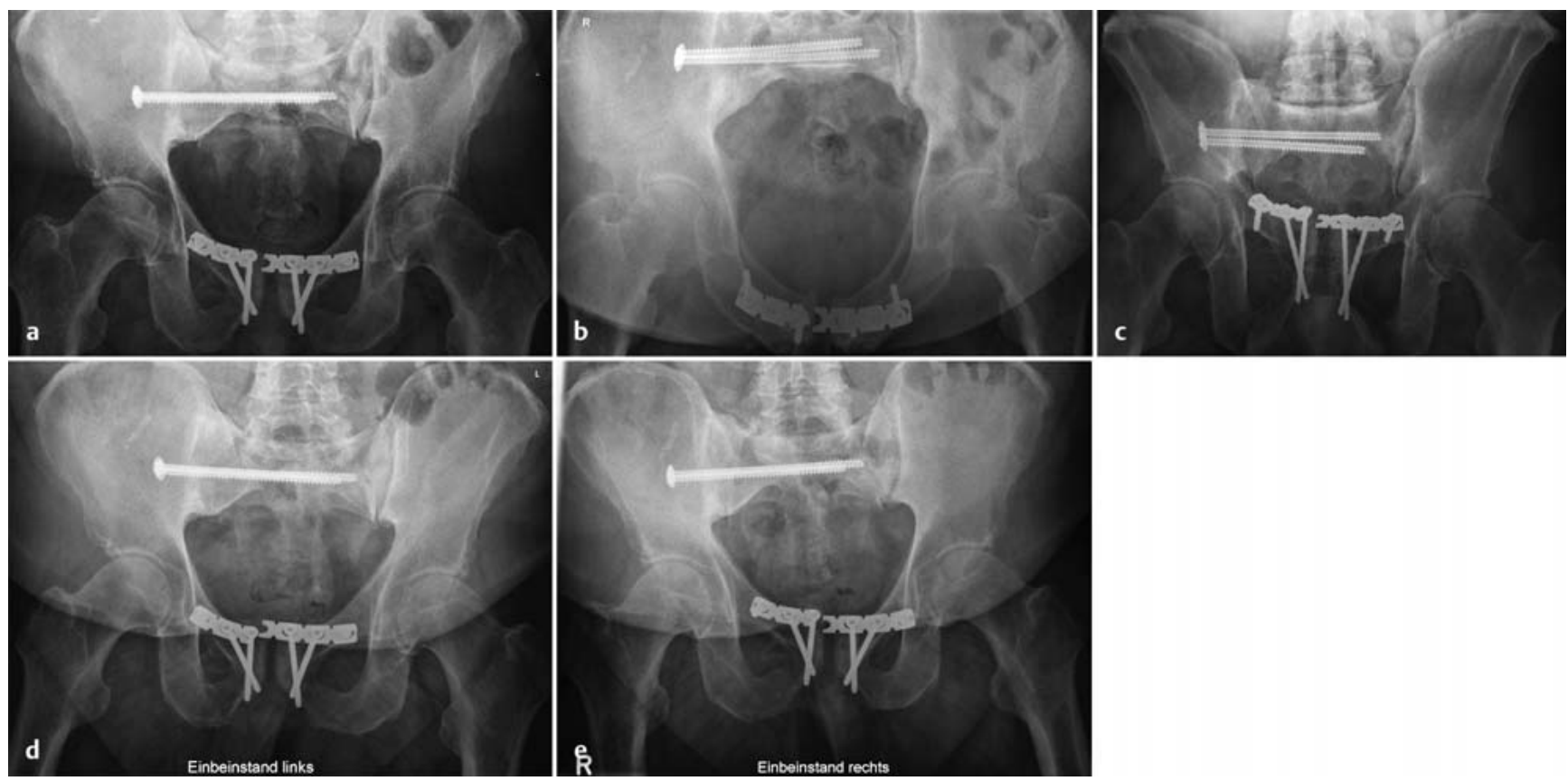

Abb. $\mathbf{5 a}$ bis e Fallbeispiel IV: Einem 53-jährigen Patienten wurde 9 Monate nach operativer Stabilisierung einer zentralen Sakrumfraktur und einer Symphysensprengung (CCF-Klassifikation C1.3 a3c5) die Metallentfernung empfohlen. Die Indikation für die empfohlene ME war insbesondere die Transfixation der ISG-Fuge. Der Patient ist beschwerdefrei und lehnte die ME sowohl am vorderen als auch hinteren Beckenring ab (a Beckenübersicht, b Inlet-Projektion, c Outlet-Projektion). Die Einbeinstandaufnahmen zeigen ein nahezu normales Bewegungsausmaß der verheilten Symphyse bei einliegender gebrochener Symphysenplatte (d Einbeinstand links, e Einbeinstand rechts).

erarbeiten, worin die vom Patienten angegebenen „implantatbedingten“ Beschwerden begründet sind. Werden die Beschwerden durch das lokal auftragende Implantat (z.B. Symphysenplatte) oder durch ein Fremdkörpergefühl bedingt, so kann der Patient von der ME profitieren. Lassen sich jedoch nativradiologische posttraumatische Veränderungen zeigen, z. B. in Form einer ISGArthrose nach ISG-Fugensprengung, so ist es bei einliegenden ISG-Schrauben oftmals schwierig, zwischen implantatbedingten Beschwerden und Arthrosebeschwerden zu unterscheiden (Abb. 4: Fallbeispiel III). Dies ist insbesondere zu beachten, wenn der Wunsch des Patienten zur ME mit großer Latenz zur definitiven Versorgung der Beckenringverletzung gestellt wird. Zumal mit größer werdendem Zeitabstand die ME technisch um ein Vielfaches anspruchsvoller werden kann aufgrund der möglichen Überknöcherung der Platten und Schraubenköpfe bzw. der Ausbildung einer kräftigen Narbenplatte. Im eigenen Kollektiv kam es hierbei im Rahmen der Entfernung von Symphysenplatten zu Blasenverletzungen bzw. im Rahmen der Entfernung von ISG-Schrauben zu Schraubenbrüchen bzw. verbleibenden Schrauben und Unterlegscheiben (Abb. 3: Fallbeispiel II). Entsprechend sollte die ME bei gesicherter Konsolidie- rung der Beckenringverletzung 6 bis 12 Monate nach Operation geplant werden $[4,27]$. Insbesondere im Bereich des hinteren Beckenrings stellt die Entfernung der i.d.R. minimalinvasiv eingebrachten ISG-Schrauben eine anspruchsvolle Operation dar, bei der oftmals eine Schnitterweiterung zum Aufsuchen und Bergen der Schrauben und Unterlegscheiben erforderlich wird. Entsprechend muss der Patient vor der ME umfassend über mögliche Komplikationen aufgeklärt werden. Im eigenen Kollektiv lag die Komplikationsrate bei ME am vorderen und hinteren Beckenring insgesamt bei $20 \%$, wohingegen bei Metallentfernungen generell über Komplikationsraten zwischen 3 und $11 \%$ berichtet wird $[20,25]$.

Eine weitere Besonderheit bei der Indikationsstellung der ME bei Patienten mit Beckenringverletzung beruht auf der Tatsache, dass oftmals im Rahmen der definitiven Versorgung eine gelenküberbrückende Osteosynthese, z.B. der Symphyse und/oder der Iliosakralfuge mittels Symphysenplatten und/oder transiliosakralen Schrauben, erforderlich war. Aufgrund der physiologischerweise auftretenden belastungsabhängigen Kräfte in der Symphyse kann durch die Osteosynthese nur für eine gewisse Zeit eine relative Stabilität erreicht werden
[23]. Daher kommt es erwartungsgemäß bei über $30 \%$ der Patienten innerhalb eines Jahres zu einer Auslockerung bzw. zu einem Bruch der einliegenden Symphysenplatte (Abb. 5: Fallbeispiel IV) [8, 16]. Im Bereich der Iliosakralfuge wird durch die Verschraubung die physiologische Gelenkbewegung im Sinne einer Arthrodese transfixiert. Das heißt, das überwiegend vertikale Bewegungsmuster der Amphiarthrose bei der Lastübertragung von der Wirbelsäule auf den Beckenring wird aufgehoben [5]. Dementsprechend besteht in der Literatur hier bislang keine Einigkeit bez. der Indikationsstellung zur Materialentfernung. Im Bereich des vorderen Beckenrings wird teilweise generell die Indikation zur ME einer einliegenden Symphysenplatte nach 6 bis 8 Monaten gestellt [4,27], teilweise wird von einer routinemäßigen ME abgesehen und eine individuelle Entscheidung gefordert [8]. Auch im eigenen Vorgehen wird im Einzelfall entschieden. Das heißt, ein gelockertes oder gebrochenes Implantat allein stellt bei asymptomatischen Patienten eher selten eine Indikation dar. Im Bereich des hinteren Beckenrings besteht teilweise die Forderung einer routinemäßigen $\mathrm{ME}$ der transiliosakralen Schrauben nach 6 bis 12 Monaten [4,14], teilweise wird die ME nur bei Komplikationen [22] bzw. unter Prüfung der individuellen Situa- 
tion [30] indiziert. Im eigenen Vorgehen wird ebenfalls im Einzelfall entschieden. Dabei wird neben einer etwaigen Schmerzsymptomatik und dem Alter des Patienten insbesondere auch das initial vorliegende Verletzungsmuster betrachtet. Das heißt, bei Beckenringverletzungen vom Typ C1.3 wird die ME tendenziell empfohlen und beim Typ C1.2 und den nativradiologischen Zeichen einer beginnenden posttraumatischen Arthrose der ISG-Fuge wird von einer ME eher Abstand genommen. Somit ist festzuhalten, dass zum jetzigen Zeitpunkt insbesondere in diesem Punkt die Grundlage für eine evidenzbasierte Empfehlung für die Metallentfernung nach Beckenringverletzungen fehlt. Die derzeitigen Empfehlungen beruhen auf individuellen Erfahrungen und Expertenmeinungen sowie biomechanischen Überlegungen. Entsprechend bedarf es einer individuellen Entscheidung nach einer intensiven und umfassenden Untersuchung und Beratung des Patienten unter Abwägung der Risiken und des Nutzens sowie des initialen Verletzungsmusters.

\section{Schlussfolgerung: „Fazit für die Praxis“}

Eine Materialentfernung nach Beckenringfraktur muss mit dem Patienten individuell und detailliert nach gründlicher klinischer und radiologischer Untersuchung abgewogen werden. Die exakte detaillierte präoperative Aufklärung der Risiken und des Nutzens der Materialentfernung ist dabei unerlässlich. Insbesondere bei posttraumatischen Veränderungen im Bereich der ISG-Fugen ist zwischen implantatbedingten Beschwerden und Arthrosebeschwerden zu unterschieden und der Patient entsprechend aufzuklären. Daher sollte bei der Entscheidungsfindung das initiale Verletzungsmuster stets mitberücksichtigt werden. Wird die Indikation zur Materialentfernung nach einer Beckenringfraktur gestellt, so handelt es sich häufig um eine technisch anspruchsvolle Operation mit einer hohen Komplikationsrate.

\section{Interessenkonflikt: Nein}

\section{Literatur}

${ }^{1}$ Abumi K, Saita M, lida T et al. Reduction and fixation of sacroiliac joint dislocation by the combined use of S1 pedicle screws and the galveston technique. Spine (Phila Pa 1976) 2000; 25: 1977-1983

2 AWMF. Leitlinie der Deutschen Gesellschaft für Unfallchirurgie: Implantatentfernung. 2008. AWMF-Leitlinien-Register Nr. 012/004

${ }^{3}$ Briem D, Rueger J, Begemann P et al. [Computer-assisted screw placement into the posterior pelvic ring: assessment of different navigated procedures in a cadaver trial]. Unfallchirurg 2006; 109: 640-646

4 Culemann $U$, Tosounidis $G$ Reilmann $H$ et al. [Injury to the pelvic ring. Diagnosis and current possibilities for treatment]. Unfallchirurg 2004; 107: 1169-1181

${ }^{5}$ Drerup B, Hierholzer E. Movement of the human pelvis and displacement of related anatomical landmarks on the body surface. J Biomech 1987; 20: 971-977

${ }^{6}$ Duchna H, Nowack U, Merget R et al. [Prospective study of the significance of contact sensitization caused by metal implants]. Zentralbl Chir 1998; 123: 1271-1276

7 Gänsslen A, Hüfner T, Krettek C. Percutaneous iliosacral screw fixation of unstable pelvic injuries by conventional fluoroscopy. Oper Orthop Traumatol 2006; 18: 225-244

8 Giannoudis P, Chalidis B, Roberts C. Internal fixation of traumatic diastasis of pubic symphysis: is plate removal essential? Arch Orthop Trauma Surg 2008; 128: 325-331

${ }^{9}$ Keating J, Werier J, Blachut P et al. Early fixation of the vertically unstable pelvis: the role of iliosacral screw fixation of the posterior lesion. J Orthop Trauma 1999; 13: 107-113

${ }^{10}$ Kregor P, Routt M jr. Unstable pelvic ring disruptions in unstable patients. Injury 1999; 30 (Suppl. 2): B19-B28

${ }^{11}$ Moss M, Bircher M. Volume changes within the true pelvis during disruption of the pelvic ring - where does the haemorrhage go? Injury 1996; 27 (Suppl. 1): S-A21-S-A23

12 Müller-Färber J. [Removal of metal in traumatology]. Unfallchirurg 2003; 106: 653-668

13 Pohlemann T, Gänsslen A, Kiessling B et al. [Determining indications and osteosynthesis techniques for the pelvic girdle]. Unfallchirurg 1992; 95: 197-209

14 Pohlemann T, Tscherne H. [Indications for surgical therapy of sacral fractures]. Chirurg 1992; 63: 884-896

15 Pohlemann T, Tscherne H, Baumgärtel F et al. [Pelvic fractures: epidemiology, therapy and long-term outcome. Overview of the multicenter study of the Pelvis Study Group]. Unfallchirurg 1996; 99: 160-167

16 Putnis S, Pearce $R$, Wali $U$ et al. Open reduction and internal fixation of a traumatic diastasis of the pubic symphysis: one-year radiological and functional outcomes. J Bone Joint Surg Br 2011; 93: 78-84

17 Ragnarsson B, Jacobsson B. Epidemiology of pelvic fractures in a Swedish county. Acta Orthop Scand 1992; 63: 297-300

${ }^{18}$ Raman $R$, Roberts C, Pape $H$ et al. Implant retention and removal after internal fixation of the symphysis pubis. Injury 2005; 36: $827-$ 831

${ }^{19}$ Remiger A, Engelhardt P. [Percutaneous iliosacral screw fixation of vertical unstable pelvic ring fractures]. Swiss Surg 1996; 2: 259263

20 Richards R, Palmer J, Clarke N. Observations on removal of metal implants. Injury 1992; 23: 25-28

${ }^{21}$ Routt M jr., Kregor P, Simonian P et al. Early results of percutaneous iliosacral screws placed with the patient in the supine position. J Orthop Trauma 1995; 9: 207-214
${ }^{22}$ Routt M jr., Simonian P, Mills W. Iliosacral screw fixation: early complications of the percutaneous technique. J Orthop Trauma 1997; 11: 584-589

${ }^{23}$ Sagi H, Papp S. Comparative radiographic and clinical outcome of two-hole and multi-hole symphyseal plating. J Orthop Trauma 2008; 22: 373-378

24 Schildhauer T, Josten C, Muhr G. Triangular osteosynthesis of vertically unstable sacrum fractures: a new concept allowing early weight-bearing. J Orthop Trauma 1998; 12: 307-314

${ }^{25}$ Schmalzried T, Grogan T, Neumeier P et al. Metal removal in a pediatric population: benign procedure or necessary evil? J Pediatr Orthop 1991; 11: 72-76

${ }^{26}$ Tile M. Acute Pelvic Fractures: I. Causation and Classification. J Am Acad Orthop Surg 1996; 4: 143-151

27 Tscherne H, Pohlemann T, Hrsg. Tscherne Unfallchirurgie: Becken und Acetabulum. Berlin, Heidelberg, New York, Tokio: Springer; 1998

${ }^{28}$ Varga E, Hearn T, Powell J et al. Effects of method of internal fixation of symphyseal disruptions on stability of the pelvic ring. Injury 1995; 26: 75-80

${ }^{29}$ Weil Y, Nousiainen M, Helfet D. Removal of an iliosacral screw entrapping the L5 nerve root after failed posterior pelvic ring fixation: a case report. J Orthop Trauma 2007; 21: 414417

${ }^{30}$ Yucel $N$, Lefering $R$, Tjardes $T$ et al. [Is implant removal after percutaneous iliosacral screw fixation of unstable posterior pelvic ring disruptions indicated?]. Unfallchirurg 2004; 107: $468-474$

Dieser Artikel basiert auf dem Artikel von Stuby FM, Gonser CE, Baron HC, Stöckle U, Badke A, Ochs BG: Implantatentfernung nach Beckenringfraktur. Unfallchirurg 2012, 115: 330-338. With permission of Springer.

PD Dr. med Fabian Maria Stuby Sektionsleiter Traumatologie Leitender OA UWCH

Dr. med. Christoph Emanuel Gonser Facharzt UWCH Dr. med. Hagen Christopher Baron Sektionsleiter AQTW Univ.-Prof. Dr. Ulrich Stöckle

Ärztlicher Direktor

PD Dr. Andreas Badke Chefarzt AQTW

Prof. Dr. med. Björn Gunnar Ochs Leitender Arzt Sektion Endoprothetik

Klinik für Unfall- und Wiederherstellungschirurgie der Eberhard Karls Universität Tübingen Berufsgenossenschaftliche

Unfallklinik Tübingen

Schnarrenbergstraße 95

72076 Tübingen

gochs@bgu-tuebingen.de 